\title{
Improvement in the Reproducibility of a Paper-based Analytical Device (PAD) Using Stable Covalent Binding between Proteins and Cellulose Paper
}

\author{
Woogyeong Hong, Seong-Geun Jeong, Gyurak Shim, Dae Young Kim, Seung Pil Pack, and Chang-Soo Lee
}

Received: 2 November 2018 / Revised: 27 November 2018 / Accepted: 28 November 2018

(C) The Korean Society for Biotechnology and Bioengineering and Springer 2018

\begin{abstract}
Paper-based analytical devices (PADs) have been widely used in many fields because they are affordable and portable. For reproducible quantitative analysis, it is crucial to strongly immobilize proteins on PADs. Conventional techniques for immobilizing proteins on PADs are based on physical adsorption, but proteins can be easily removed by weak physical forces. Therefore, it is difficult to ensure the reproducibility of the analytical results of PADs using physical adsorption. To overcome this limitation, in this study, we showed a method of covalent binding of proteins to cellulose paper. This method consists of three steps, which include periodate oxidation of paper, the formation of a Schiff base, and reductive amination. We identified aldehyde and imine groups formed on paper using FT-IR analysis. This covalent bonding approach enhanced the binding force and binding capacity of proteins. We confirmed the activity of an immobilized antibody through a sandwich immunoassay. We expect that this immobilization method will contribute to the commercialization of PADs with high reproducibility and sensitivity.
\end{abstract}

Woogyeong Hong, Seong-Geun Jeong, Gyurak Shim, Chang-Soo Lee* Department of Chemical Engineering and Applied Chemistry, Chungnam National University, Korea

Tel: +82-42-821-5896; Fax: +82-42-822-8995

E-mail: rhadum@cnu.ac.kr

Dae Young Kim

New Drug Development Center, Osong Medical Innovation Foundation, Korea

Seung Pil Pack*

Department of Biotechnology and Bioinformatics, Korea University, Korea

Tel: +82-44-860-1419; Fax: +82-44-860-1598

E-mail:spack@korea.ac.kr
Keywords: cellulose, immobilization, periodate oxidation, covalent binding, protein

\section{Introduction}

In recent years, easy-to-use and low-cost diagnostic devices have been developed for applications in the developing world [1-7] this is because clinical diagnosis is the most important step in the treatment of disease. Current clinical diagnoses that are available in the developed world require expensive reagents and sophisticated equipment. Therefore, in the developing world, where access to clinical diagnosis is limited, patients can suffer because of the cost of personal diagnosis [8]. Therefore, to overcome this problem, it is very important to develop a point-of-care (POC) device. A POC device can provide accurate and rapid results for diagnosis at a site of patient care. Ideal POC devices are required to be "ASSURED", a criteria defined by the World Health Organization (WHO) $[9,10]$. "ASSURED" devices must be affordable, sensitive, specific, user friendly, rapid and robust, equipment free, and deliverable, as outlined by the WHO criteria. These criteria are an essential guideline for future diagnostic devices. To achieve these criteria, especially affordability, it is crucial to select inexpensive materials for the fabrication of PADs.

Paper has been used extensively as an ideal platform for portable diagnostic devices that are particularly useful in limited-resource settings and the developing world [10-14]. Paper facilitates the immobilization of detection materials and the diffusion of the analyte because the paper has a porous structure [15]. Additionally, paper can be easily patterned by conventional photolithography processes [16], inkjet printing [17], wax printing [18], or screen printing 
methods [19] for mass production. Moreover, paper is abundant and affordable. Due to the properties of paper, paper-based analytical devices (PAD) have many advantages. The advantages of PADs are simple fabrication, low sample and reagent consumption, rapid analysis, simple operation, disposability, and portability [10,20-23]. Because of these characteristics, PADs are currently used in a wide range of applications, such as environmental monitoring [12,24,25], medical diagnosis $[2,10,12,26]$, and food safety monitoring $[10,12]$.

For detecting and quantifying biomolecules, effective immobilization of these biomolecules on solid surfaces is an important step in biosensor fabrication. To date, conventional techniques for biomolecule immobilization on paper-based sensors are based on physical adsorption [21]. However, biomolecules are physically bound to paper fibers. Physically adsorbed biomolecules can be easily removed due to weak physical forces, such as electrostatic, van der Waals and hydrophobic interactions between biomolecules and paper. Therefore, the physical adsorption of biomolecules cannot always assure reproducible results [27]. Wei Shen's group has shown that approximately $40 \%$ of antibodies adsorbed on paper can be desorbed from the paper substrate by washing [28]. For more sensitive and quantitative bioanalysis, it is crucial to immobilize biomolecules on paper-based sensors using immobilization via covalent bonds. To overcome this problem, we present a method of covalent binding of proteins to paper for a paper-based sandwich enzyme-linked immunosorbent assay (ELISA) to detect MERS-CoV.

\section{Materials and Methods}

\subsection{Reagents and materials}

Sodium periodate $\left(\mathrm{NaIO}_{4}\right.$, ACS reagent grade, $\left.\geq 99.8 \%\right)$, sodium cyanoborohydride $\left(\mathrm{NaCNBH}_{3}\right.$, reagent grade), albumin-fluorescein isothiocyanate conjugate (FITC-BSA) and bovine serum albumin (BSA) were purchased from Sigma-Aldrich (St. Louis, MO). Middle East respiratory syndrome coronavirus (MERS-CoV) capture antibody (D5) was obtained from the New Drug Development Center of Osong Medical Innovation Foundation(KBioHealth) under the permission of Korea Centers for Disease Control \& Prevention (KCDC). Horseradish peroxidase (HRP)-labeled anti-6X His-tagged antibody was purchased from Abcam (Cambridge, UK). MERS-CoV antigen was purchased from Sino Biological, Inc. (Beijing, China). 3,3',5,5'Tetramethylbenzidine (TMB) substrate solution was purchased from Thermo Scientific (MA, USA). Whatman No. 1 chromatography paper was obtained from GE Healthcare Worldwide (Pudong Shanghai, China). Coupling buffer for protein immobilization was PBS buffer (10 $\mathrm{mM}$ phosphate buffered saline, $\mathrm{pH}$ 7.4). Blocking buffer was PBS buffer containing $1 \%$ BSA to block residual reactive sites. Washing buffer was PBS buffer containing $0.05 \%$ Tween-20 to minimize nonspecific adsorption.

\subsection{Preparation and fabrication of paper-based analytical devices (PADs)}

PADs were fabricated by a wax patterning process. The pattern for wax patterning was designed using Adobe Illustrator CS5. The designed test area was a circle with a diameter of $4 \mathrm{~mm}$. The designed pattern was printed on chromatography paper (Whatman No. 1 chromatography paper) using a wax printer (Xerox Colorqube 8870). Subsequently, the printed paper was heated at $160^{\circ} \mathrm{C}$ for $8 \mathrm{sec}$ using a laminator (PhotoLami-350R6, Hyundai Office Co., Ltd., Republic of Korea) and then naturally cooled at room temperature. A laminator is able to control the rate of lamination by the rotation rate of rollers [29]. Consequently, wax can melt and spread on the paper to form a hydrophobic wax barrier on the paper. These wax patterning fabrication processes can be completed within $5 \mathrm{~min}$.

\subsection{Covalent immobilization of antibodies on paper}

The process of the covalent binding of proteins on paper consists of periodate oxidation of paper, the formation of a Schiff base, and reductive amination of the paper. First, $10 \mu \mathrm{L} \mathrm{NaIO}_{4}(0.5 \mathrm{~mol} / \mathrm{L})$ was spotted on each test area and allowed to react at room temperature to produce an aldehyde group in the dark for $30 \mathrm{~min}$. After oxidation, the test areas were washed twice. We added $10 \mu \mathrm{L}$ ultrapure water to the top of the test areas and washed the test areas by pressing the bottom of the areas against a piece of blotting paper [30]. This washing method was used consistently and acquiescently in this experiment. Second, for covalent binding with proteins, we added $3 \mu \mathrm{L}$ BSA $(1-10 \mathrm{mg} / \mathrm{mL}$ in PBS) to each aldehyde group-formed paper and allowed it to incubate for $30 \mathrm{~min}$ at room temperature. Subsequently, unbound proteins were washed twice with $10 \mu \mathrm{L}$ ultrapure water as described above. We then treated each test area with $10 \mu \mathrm{L} \mathrm{NaCNBH}_{3}(1.6 \mathrm{mg} / \mathrm{mL})$ to obtain stable covalent bonds (secondary amines). The BSA-immobilized paper was then washed twice with $10 \mu \mathrm{L}$ washing buffer (Fig. 1).

To confirm that the protein was immobilized on the paper, the analysis was carried out in two steps. First, using $3 \mu \mathrm{L}$ BSA ( $5 \mathrm{mg} / \mathrm{mL}$ in PBS), the FT-IR spectra of the test area for each reaction step were obtained and measured on a VERTEX 80v FT-IR spectrometer. Second, using $3 \mu \mathrm{L}$ FITC- BSA (1-10 $\mathrm{mg} / \mathrm{mL}$ in PBS), we immobilized proteins on paper and then washed them sufficiently. The purpose of this experiment was to confirm the binding force of covalently immobilized proteins. This result was compared 

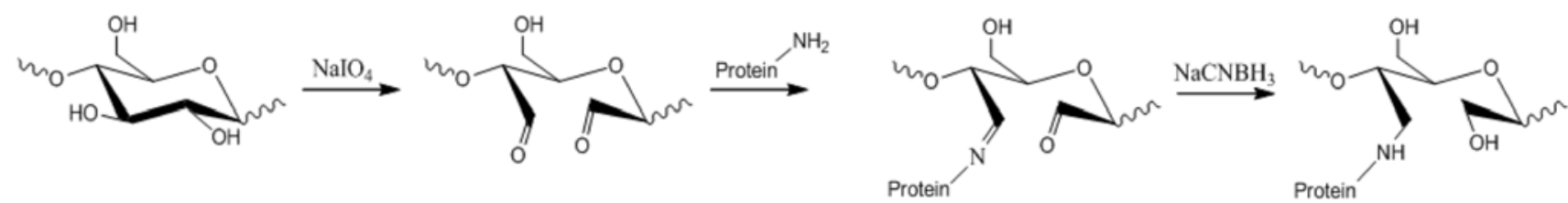

Fig. 1. A schematic representation of the process for the covalent immobilization of protein on paper as follows: (1) original paper consists of pure cellulose; (2) an aldehyde group is formed by oxidation with sodium periodate; (3) the aldehyde group reacts with the amino group of a protein to form a reversible Schiff base; and (4) Schiff bases and unreacted aldehyde groups are then reduced using $\mathrm{NaCNBH}_{3}$

with the protein immobilization method using physical adsorption. Fluorescence images of the washed paper were obtained using a fluorescence microscope (TE2000, Nikon, Japan) equipped with a CCD camera (CoolSNAP cf, Photometrics, USA). The fluorescence intensity of the image was analyzed quantitatively using ImageJ software.

\subsection{Paper-based sandwich ELISA for MERS-CoV antigen detection}

For covalent binding of protein, $3 \mu \mathrm{L}$ MERS-CoV capture antibody solution $(80 \mu \mathrm{g} / \mathrm{mL})$ was added to the aldehyde group-formed paper area. Covalent binding was performed as described above. After the covalent binding process, $10 \mu \mathrm{L}$ of blocking buffer solution was added and incubated for $15 \mathrm{~min}$ to block nonspecific binding to the test areas. Three microliters of MERS-CoV antigen (1.22 $\mathrm{fM}$ to $7.6 \mu \mathrm{M})$ was added to the test area on the antibodyimmobilized paper and incubated for $3 \mathrm{~min}$. We washed the test area twice with $10 \mu \mathrm{L}$ washing buffer to remove unbound proteins from the test areas. Next, we added $3 \mu \mathrm{L}$ HRP-labeled anti-6X His-tagged antibody (1:5000 dilution of stock antibody solution in $0.05 \%(\mathrm{v} / \mathrm{v})$ Tween-20 in PBS) and incubated for $3 \mathrm{~min}$. Subsequently, after washing as described above, $3 \mu \mathrm{L}$ TMB substrate solution was added to each test area. After $8 \mathrm{~min}$, the test areas were scanned with a document scanner (JP/Stylus Office TX600FW scanner, EPSON Corp.), and the image was analyzed quantitatively using ImageJ software.

\section{Results and Discussion}

The process of protein immobilization by covalent binding on pure cellulose paper proceeded as follows. (1) Periodate oxidation is a highly specific reaction to convert a 1,2dihydroxyl (glycol) group to an aldehyde (-CHO) group in pure cellulose paper using sodium periodate as the oxidizing agent [31-33]. (2) The aldehyde group formed on the paper reacted with the amino $\left(-\mathrm{NH}_{2}\right)$ group of the protein to form a reversible Schiff base; [34] (3) The Schiff base and the unreacted aldehyde group were reacted with $\mathrm{NaCNBH}_{3}$ and reduced by a reductive amination process

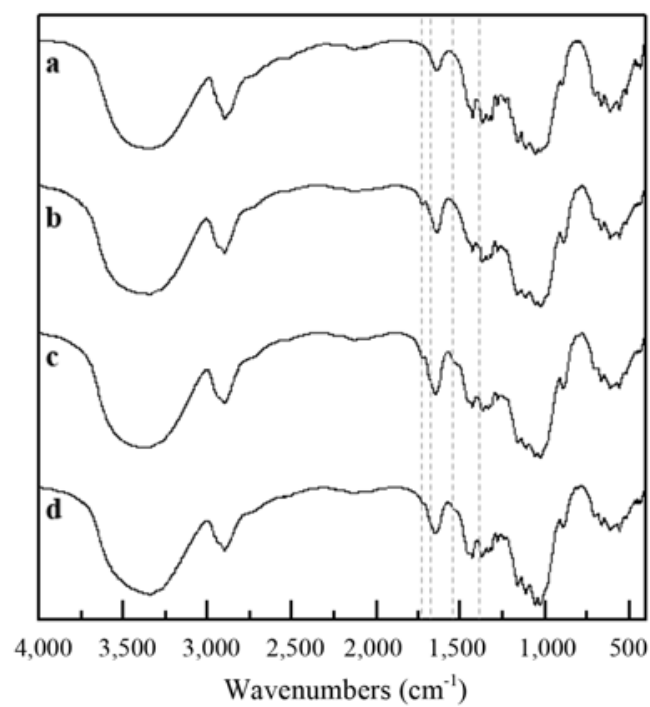

Fig. 2. FT-IR spectra of (a) bare cellulose, (b) periodate-oxidized cellulose, (c) BSA-immobilized cellulose, and (d) $\mathrm{NaCNBH}_{3}-$ treated cellulose.

[35]. The Schiff base was rapidly hydrolyzed in an aqueous solution [36] (Fig. 1).

We confirmed the aldehyde groups and Schiff bases formed on the paper by Fourier transform infrared spectroscopy (FT-IR spectroscopy). The FT-IR spectra of bare paper (spectrum a), periodate-oxidized paper (spectrum b), BSA-immobilized paper (spectrum c) and reduced paper (spectrum d) are shown in Fig. 2. The periodate-oxidized paper (spectrum b) showed new absorption band peaks at $1729 \mathrm{~cm}^{-1}$ and $1387 \mathrm{~cm}^{-1}$, which was not present in bare paper (spectrum a). These absorption peaks correspond to carbonyl groups $(\mathrm{C}=\mathrm{O})$ and $\mathrm{C}-\mathrm{H}$ bonds of aldehyde groups, respectively. This result shows that aldehyde groups were successfully formed by periodate oxidation of the paper. Subsequently, we confirmed the presence of bound BSA after immobilizing BSA on periodate-oxidized paper. New absorption band peaks appeared at $1677 \mathrm{~cm}^{-1}$ and $1540 \mathrm{~cm}^{-1}$ on BSA-immobilized paper (spectrum c) and reduced paper (spectrum d). These absorption peaks correspond to the imine group $(\mathrm{C}=\mathrm{N})$ of the Schiff base and the combination of $\mathrm{N}-\mathrm{H}$ bending and $\mathrm{C}-\mathrm{N}$ stretching, respectively. This 
A

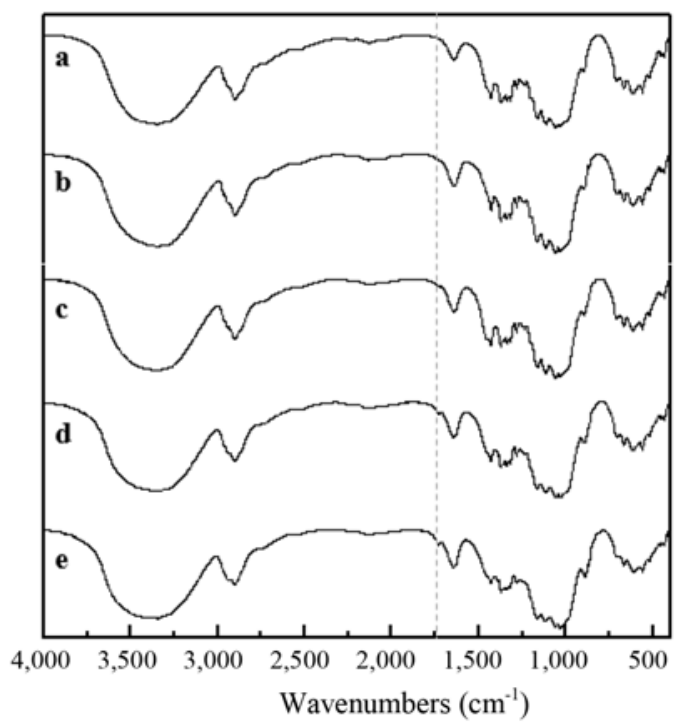

B
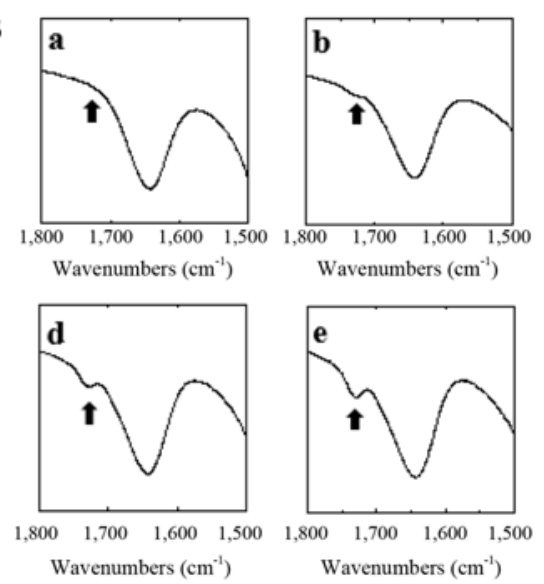

Fig. 3. FT-IR spectra of oxidized cellulose under different oxidation conditions. (a) Bare cellulose; (b) $0.5 \mathrm{~h}$ oxidation time; (c) $1 \mathrm{~h}$ oxidation time; (d) $3 \mathrm{~h}$ oxidation time; and (e) $6 \mathrm{~h}$ oxidation time.

result shows that BSA was successfully immobilized on the paper.

To investigate the appropriate reaction time for the formation of aldehyde groups by oxidation, the degree of oxidation of the paper according to the oxidation reaction time was measured by FT-IR. Fig. 3A shows the full range of FT-IR spectra, and Fig. 3B shows a magnified area of the spectra from $1800 \mathrm{~cm}^{-1}$ to $1500 \mathrm{~cm}^{-1}$ for observing the carbonyl bands. The reaction time (oxidation time) with sodium periodate was $30 \mathrm{~min}$ to $6 \mathrm{~h}$. These spectral data show that the absorption peak intensity at $1729 \mathrm{~cm}^{-1}$ increased with increasing oxidation time. The absorption peak at $1729 \mathrm{~cm}^{-1}$ corresponds to the carbonyl band $(\mathrm{C}=\mathrm{O})$ of the aldehyde group. This indicates that the paper gradually oxidized with increasing reaction time.

However, as the oxidation time increased, periodate oxidation drastically changed the cellulose morphology.

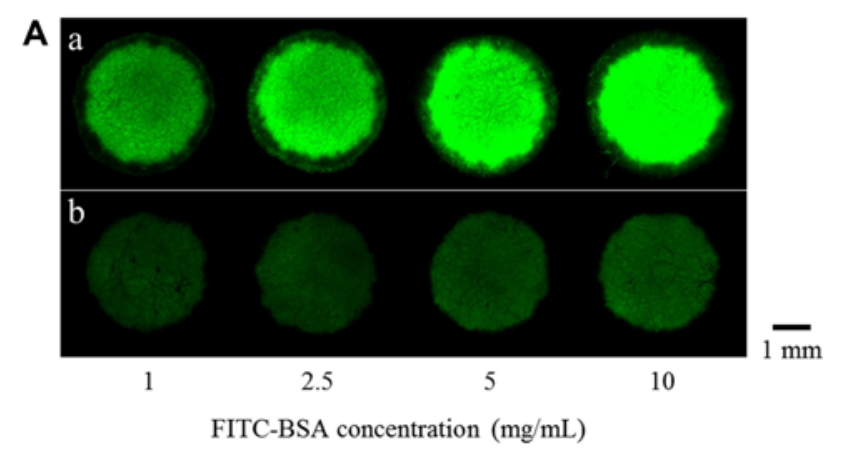

B

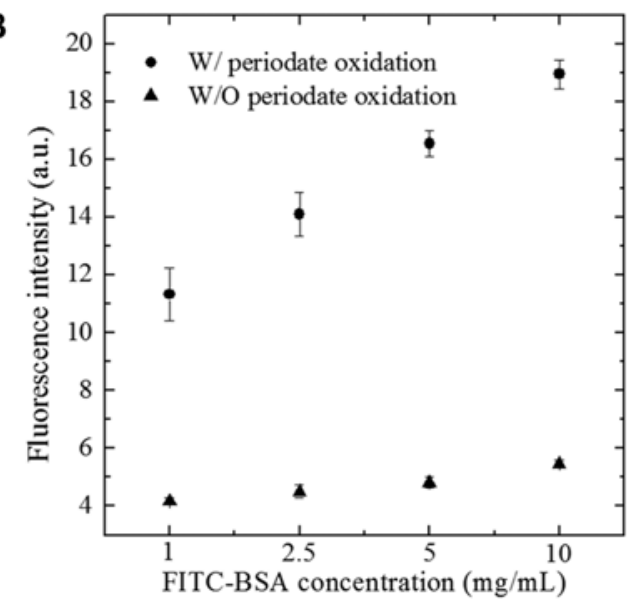

Fig. 4. Fluorescence image of the FITC-BSA-immobilized paper zone through physical adsorption and covalent binding after 10 washes. (A) Fluorescence images results from $1 \mathrm{mg} / \mathrm{mL}$ to $10 \mathrm{mg} / \mathrm{mL}$ of FITC-BSA. (a) Covalent bonding using periodate oxidized paper; (b) physical adsorption without periodate oxidized paper. (B) Effects of covalent binding on fluorescence intensity, where $n=6$ for each point.

The stiffness of the cellulose fibers was reduced by the ring-opening of glucose, and changes in the fiber dimensions and the formation of interfibrillar and intrafibrillar hemiacetal crosslinks were also observed. This contributed to the observed morphological changes [37,38]. Thus, when the degree of oxidation increased, the cellulose became more compact. Therefore, we determined that a periodate oxidation time of $30 \mathrm{~min}$ was able to form aldehyde groups on the paper without destructive changes to the paper.

To investigate the binding force of covalently bound proteins on the periodate-oxidized paper, we performed washing experiments. It is crucial to strongly immobilize analyte proteins on the biosensor for quantification assays that are reproducible and sensitive. Conventional techniques for immobilizing proteins on paper-based sensors are based on physical adsorption. Therefore, to compare covalent binding with physical adsorption, we immobilized FITCBSA on paper using the covalent binding method with periodate oxidation (a in Fig. 4A) and physical adsorption without periodate oxidation (b in Fig. 4A). FITC-BSA 
was immobilized at various concentrations $(1 \mathrm{mg} / \mathrm{mL}$ to $10 \mathrm{mg} / \mathrm{mL}$ ). After 10 washes with FITC-BSA-immobilized paper, the fluorescence intensity was compared. The values on the graph are represented by the signal-to-noise ratio, and the larger the signal-to-noise ratio is, the greater the signal size relative to the background noise.

Fig. 4A shows a clear enhancement in fluorescence intensity, indicating efficient immobilization of FITC-BSA on the periodate-oxidized paper. Each image is a fluorescence image of paper areas washed 10 times after protein immobilization. Fig. 4Aa is the fluorescence image obtained by covalent binding of FITC-BSA to paper using periodate oxidation. Fig. 4Ab shows the fluorescence image obtained by physically adsorbing FITC-BSA without periodate oxidation. The fluorescence intensity of paper using the covalent binding method was significantly greater than the fluorescence intensity of paper using the physical adsorption method, even though the same concentration of FITC-BSA was immobilized. To analyze and compare fluorescence intensity quantitatively, we measured the intensity using ImageJ software (Fig. 4B). In the case of covalent binding of FITC-BSA, the fluorescence intensity increased linearly with increasing protein concentration. The fluorescence intensity increased when the amount of FTIC-BSA immobilized on the paper increased. This result indicates that the amount of protein immobilized on the paper can be quantified according to the concentration of the protein. However, when FITC-BSA was physically adsorbed, the fluorescence intensity increased slightly when the concentration increased. This indicates that when the fluorescence intensity increased, the amount of FTIC-BSA adsorbed on the paper also increased, but the fluorescence intensity only slightly increased. This is because unbound or physically adsorbed proteins on the paper were easily washed away through several washing processes. Additionally, pure cellulose has a relatively low capacity for protein adsorption compared to that of periodate-oxidized paper.

Therefore, fluorescence image analysis revealed that the covalent binding of proteins is a more effective method than physical adsorption because of the greater amount of captured protein on periodate-oxidized paper. This indicates that the protein binding capacity of periodate-oxidized paper was improved compared with that of pure cellulose paper. This result also shows that when FITC-BSA is covalently immobilized on the periodate-oxidized paper, the protein binds more strongly to the paper than through physical adsorption. In addition, the stability of immobilized antibodies on a paper surface has been studied by Fang Zeng's group [39]. Their results showed that fluorescence intensities using covalent binding of antibody molecules on paper were reduced by $25 \%$ of the initial values. Compared to the intensities for covalent binding, the fluorescence intensities using physical adsorption of antibody molecules on paper were reduced by $80 \%$ of the initial values. This indicates that antibody stability is much greater when using covalent binding of antibodies. Physically adsorbed biomolecules can be easily removed due to the weak physical forces between biomolecules and paper. The instability of adhesion results in the heterogeneity of the amount of adsorbed biomolecules before the assay. Due to the heterogeneity of the amount of adsorbed biomolecules, it is difficult to ensure the reproducibility of analytical results of biosensors using physical adsorption. Thus, covalent binding using periodate-oxidized paper could enhance the binding capacity and binding ability of protein on paper, which could enhance the sensitivity and reproducibility of paper-based analytical devices.

Based on these experiments, we performed a paper-based sandwich ELISA to confirm the activity of the proteins immobilized on paper. We used a colorimetric method so that the results of the paper-based sandwich ELISA could be interpreted without plate readers or fluorescence scanners. Fig. 5A shows a schematic diagram of a paper-based sandwich ELISA for the detection of MERS CoV. The MERS-CoV antigen was used as a model analyte. The MERS-CoV capture antibody (D5) and HRP-labeled anti6X His-tagged antibody were used as primary and secondary antibodies. The MERS-CoV capture antibody was covalently immobilized on the paper through an aldehyde group formed by periodate oxidation. Because the MERS-CoV capture antibody is specific for the MERS-CoV antigen, it interacts with the MERS-CoV antigen to form an antibodyantigen complex. To detect the MERS-CoV antigen attached to the MERS-CoV capture antibody, the HRP-labeled anti$6 \mathrm{X}$ His-tagged antibody was added to the paper. The HRPlabeled anti-6X His-tagged antibody recognized the MERS$\mathrm{CoV}$ antigen and formed a sandwich structure. Because of the presence of the Histidine tag in the MERS-CoV antigen, it binds to HRP-labeled anti-6X His-tagged antibodies that recognize histidine. After addition of the TMB substrate, the HRP enzyme in the sandwich structure reacts with TMB substrate to form the color changes from transparent to blue [40], indicating the presence of the MERS-CoV antigen.

Fig. 5B shows an image of paper areas as a result of the sandwich ELISA for the detection of MERS-CoV. The paper-based sandwich ELISA produced different color signals for the MERS-CoV antigen with different concentrations. The intensity of the blue color was developed by the HRP-labeled antibody. This intensity was proportional to the number of detected MERS-CoV antigens. This result indicates that as the concentration of the antigen increased, the amount of antigen recognized by the MERS-CoV capture antibody bound to the paper increased. To analyze the color intensity quantitatively, the paper was scanned 


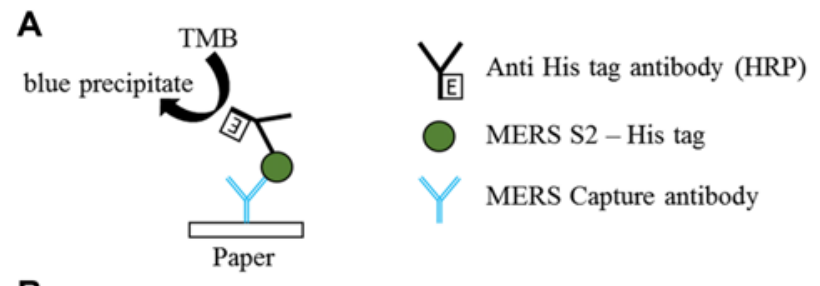

B
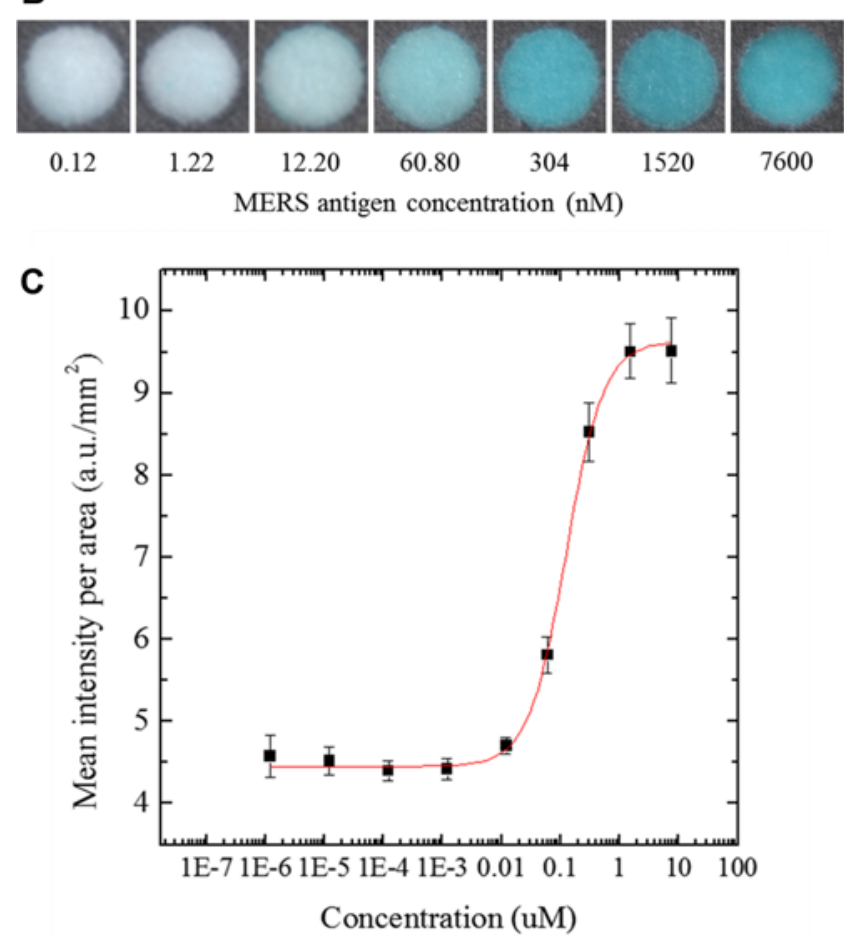

Fig. 5. Paper-based sandwich ELISA for the MERS antigen. (A) A schematic of the paper-based sandwich ELISA for the MERS antigen. (B) Images of the sandwich ELISA results from $1.22 \mathrm{fM}$ to $7.6 \mu \mathrm{M}$ of MERS antigen. (C) Calibration plot for the mean intensity of the color produced by the enzymatic reaction in the sandwich ELISA assay versus the concentration of the MERS antigen. Each datapoint is the mean of eight replicates $(n=5)$, and the error bars represent the standard deviations of the measurements.

with a document scanner, and the color intensity of each test area was analyzed using ImageJ software. Concentration versus relative intensity plots of the paper-based sandwich ELISA are shown in Fig. 5C. The standard curve was made by a standard four-parameter logistic regression, and a sigmoidal curve was obtained. The plot obtained from the paper-based sandwich ELISA provides an approximate quantitative estimation of bound MERS-CoV antigen by the color intensity. We determined the limit of detection of the MERS-CoV antigen using a paper-based sandwich ELISA. The limit of detection of the MERS-CoV antigen was approximately $10.4 \mathrm{nM}$, which is three times the standard deviation of the control.

These results indicate that the MERS-CoV capture antibody bound to the paper by covalent bonds had an antigen-recognition activity. Through covalently immobilized antibodies, we have successfully performed a paper-based sandwich ELISA, which enabled quantitative analysis of the target protein. We expect that not only antibodies but also other biomolecules can retain their activity when covalently immobilized. Therefore, these results show that PADs can be applied to other applications in the field of point-of-care diagnostic devices.

\section{Conclusion}

In conclusion, we successfully performed protein immobilization by covalent binding on pure cellulose paper. We immobilized antibodies on periodate-oxidized paper through Schiff base formation and a reductive amination process. We confirmed that the covalent bonds formed on the paper had high binding forces of the antibody. In addition, we successfully performed a paper-based sandwich ELISA. We showed that paper-based sandwich ELISA can be used to detect and quantify the MERS-CoV antigen using an antibody conjugated to HRP to produce a colorimetric readout. This result indicated that the covalently immobilized antibody retained antibody activity. Based on this result, covalent immobilization of proteins using periodate-oxidized paper could be a promising route for attaching various amine-containing biomolecules, such as bacteria, enzymes, and DNA, on paper surfaces. Thus, we expect that this covalent immobilization method will be useful for paperbased analytical devices (PADs) for quantitative assays with high reproducibility and sensitivity.

\section{Acknowledgements}

This work was supported by the Global Research Laboratory (NRF-2015K1A1A2033054) and the Basic Science Research Program (NRF-2017M3A9B6062989) through the National Research Foundation of Korea (NRF) funded by the Ministry of Science and ICT (Information and Communication Technologies). This work was supported by the Commercializations Promotion Agency for R\&D Outcomes(COMPA) funded by the Ministry of Science and ICT (Information and Communication Technologies) (2018K000148).

\section{References}

1. Weigl, B., G. Domingo, P. Labarre, and J. Gerlach (2008) Towards non- and minimally instrumented, microfluidics-based diagnostic devices. Lab on a Chip. 8: 1999-2014.

2. Martinez, A. W., S. T. Phillips, G. M. Whitesides, and E. Carrilho (2010) Diagnostics for the developing world: microfluidic paper- 
based analytical devices. Analytical Chemistry 82: 3-10.

3. Mabey, D., R. W. Peeling, A. Ustianowski, and M. D. Perkins (2004) Diagnostics for the developing world. Nature Reviews Microbiology 2: 231-240.

4. Mao, X. and T. J. Huang (2012) Microfluidic diagnostics for the developing world. Lab on a Chip. 12: 1412-1416.

5. Yang, J. M., K. R. Kim, and C. S. Kim (2018) Biosensor for Rapid and Sensitive Detection of Influenza Virus. Biotechnol. Bioproc. E. 23: 371-382.

6. Zhao, Z., J. Zhang, M. L. Xu, Z. P. Liu, H. Wang, M. Liu, Y. Y. Yu, L. Sun, H. Zhang, and H. Y. Wu (2016) A rapidly new-typed detection of norovirus based on F0F1-ATPase molecular motor biosensor. Biotechnol. Bioproc. E. 21: 128-133.

7. Sobhan, A., J. H. Oh, M. K. Park, S. W. Kim, C. Park, and J. Lee (2018) Single walled carbon nanotube based biosensor for detection of peanut allergy-inducing protein ara h1. Korean Journal of Chemical Engineering 35: 172-178.

8. von Lode, P. (2005) Point-of-care immunotesting: approaching the analytical performance of central laboratory methods. Clinical Biochemistry 38: 591-606.

9. Peeling, R. W., K. K. Holmes, D. Mabey, and A. Ronald (2006) Rapid tests for sexually transmitted infections (STIs): the way forward. Sexually Transmitted Infections 82 Suppl 5: v1-6.

10. Yetisen, A. K., M. S. Akram, and C. R. Lowe (2013) Paper-based microfluidic point-of-care diagnostic devices. Lab on a Chip. 13: 2210-2251

11. Sackmann, E. K., A. L. Fulton, and D. J. Beebe (2014) The present and future role of microfluidics in biomedical research. Nature 507: 181-189.

12. Cate, D. M., J. A. Adkins, J. Mettakoonpitak, and C. S. Henry (2015) Recent developments in paper-based microfluidic devices. Analytical Chemistry 87: 19-41.

13. Han, S. I., K. S. Hwang, R. Kwak, and J. H. Lee (2016) Microfluidic paper-based biomolecule preconcentrator based on ion concentration polarization. Lab on a Chip. 16: 2219-2227.

14. Yu, L. and Z. Z. Shi (2015) Microfluidic paper-based analytical devices fabricated by low-cost photolithography and embossing of Parafilm(R). Lab on a Chip. 15: 1642-1645.

15. Lee, C. H., L. Tian, and S. Singamaneni (2010) Paper-based SERS swab for rapid trace detection on real-world surfaces. $A C S$ Applied Materials \& Interfaces 2: 3429-3435.

16. Dungchai, W., O. Chailapakul, and C. S. Henry (2009) Electrochemical detection for paper-based microfluidics. Analytical Chemistry 81: 5821-5826.

17. Lessing, J., A. C. Glavan, S. B. Walker, C. Keplinger, J. A. Lewis, and G. M. Whitesides (2014) Inkjet printing of conductive inks with high lateral resolution on omniphobic " $\mathrm{R}(\mathrm{F})$ paper" for paper-based electronics and MEMS. Advanced Materials 26: 4677-4682.

18. Carrilho, E., A. W. Martinez, and G. M. Whitesides (2009) Understanding wax printing: a simple micropatterning process for paper-based microfluidics. Analytical Chemistry. 81: 7091-7095.

19. Noor, M. O., A. Shahmuradyan, and U. J. Krull (2013) Paperbased solid-phase nucleic acid hybridization assay using immobilized quantum dots as donors in fluorescence resonance energy transfer. Analytical Chemistry 85: 1860-1867.

20. Parolo, C. and A. Merkoci (2013) Paper-based nanobiosensors for diagnostics. Chemical Society Reviews 42: 450-457.

21. Jeong, S. G., J. Kim, J. O. Nam, Y. S. Song, and C. S. Lee (2013) Paper-based analytical device for quantitative urinalysis. International Neurourology Journal 17: 155-161.

22. Taudte, R. V., A. Beavis, L. Wilson-Wilde, C. Roux, P. Doble, and L. Blanes (2013) A portable explosive detector based on fluorescence quenching of pyrene deposited on coloured wax- printed muPADs. Lab on a Chip. 13: 4164-4172.

23. Jeong, S. G., J. Kim, S. H. Jin, K. S. Park, and C. S. Lee (2016) Flow control in paper-based microfluidic device for automatic multistep assays: A focused minireview. Korean Journal of Chemical Engineering 33: 2761-2770.

24. Yang, Y., E. Noviana, M. P. Nguyen, B. J. Geiss, D. S. Dandy, and C. S. Henry (2017) Paper-based microfluidic devices: Emerging themes and applications. Analytical Chemistry 89: 71-91.

25. Meredith, N. A., C. Quinn, D. M. Cate, T. H. Reilly, 3rd, J. Volckens, and C. S. Henry (2016) Paper-based analytical devices for environmental analysis. The Analyst 141: 1874-1887.

26. Yamada, K., T. G. Henares, K. Suzuki, and D. Citterio (2015) Paper-based inkjet-printed microfluidic analytical devices. Angewandte Chemie 54: 5294-5310.

27. Credou, J., H. Volland, J. Dano, and T. Berthelot (2013) A onestep and biocompatible cellulose functionalization for covalent antibody immobilization on immunoassay membranes. Journal of Materials Chemistry B. 1: 3277-3286.

28. Jarujamrus, P., J. Tian, X. Li, A. Siripinyanond, J. Shiowatana, and W. Shen (2012) Mechanisms of red blood cells agglutination in antibody-treated paper. The Analyst 137: 2205-2210.

29. Jeong, S. G., S. H. Lee, C. H. Choi, J. Kim, and C. S. Lee (2015) Toward instrument-free digital measurements: a three-dimensional microfluidic device fabricated in a single sheet of paper by doublesided printing and lamination. Lab on a Chip. 15: 1188-1194.

30. Cheng, C. M., A. W. Martinez, J. Gong, C. R. Mace, S. T. Phillips, E. Carrilho, K. A. Mirica, and G. M. Whitesides (2010) Paperbased ELISA. Angewandte Chemie 49: 4771-4774.

31. Kim, U. J., S. Kuga, M. Wada, T. Okano, and T. Kondo (2000) Periodate oxidation of crystalline cellulose. Biomacromolecules 1: 488-492.

32. Maekawa, E., and T. Koshijima (1984) Properties of 2,3dicarboxy cellulose combined with various metallic ions. Journal of Applied Polymer Science 29: 2289-2297.

33. Bruneel, D. and E. Schacht (1993) Chemical modification of pullulan: 1. Periodate oxidation. Polymer 34: 2628-2632.

34. Alonso, D., M. Gimeno, J. D. Sepulveda-Sanchez, and K. Shirai (2010) Chitosan-based microcapsules containing grapefruit seed extract grafted onto cellulose fibers by a non-toxic procedure. Carbohydrate Research 345: 854-859.

35. Abdel-Magid, A. F., K. G. Carson, B. D. Harris, C. A. Maryanoff, and R. D. Shah (1996) Reductive Amination of Aldehydes and Ketones with Sodium Triacetoxyborohydride. Studies on Direct and Indirect Reductive Amination Procedures(1). The Journal of Organic Chemistry 61: 3849-3862.

36. Cordes, E. H. and W. P. Jencks (1962) On the mechanism of schiff base formation and hydrolysis. Journal of the American Chemical Society 84: 832-837.

37. Lindh, J., D. O. Carlsson, M. Stromme, and A. Mihranyan (2014) Convenient one-pot formation of 2,3-dialdehyde cellulose beads via periodate oxidation of cellulose in water. Biomacromolecules 15: 1928-1932.

38. Potthast, A., M. Kostic, S. Schiehser, P. Kosma, and T. Rosenau (2007) Studies on oxidative modifications of cellulose in the periodate system: Molecular weight distribution and carbonyl group profiles. Holzforschung 61: 662-667.

39. Wang, S., L. Ge, X. Song, M. Yan, S. Ge, J. Yu, and F. Zeng (2012) Simple and covalent fabrication of a paper device and its application in sensitive chemiluminescence immunoassay. The Analyst 137: 3821-3827.

40. Josephy, P. D. (1985) Oxidative activation of benzidine and its derivatives by peroxidases. Environmental Health Perspectives 64: 171-178.

Publisher's Note Springer Nature remains neutral with regard to jurisdictional claims in published maps and institutional affiliations. 